\title{
Image Selection in Photogrammetric Multi-View Stereo Methods for Metric and Complete 3D Reconstruction
}

\author{
Ali Hosseininaveh Ahmadabadian*a, Stuart Robson ${ }^{\mathrm{a}}$, Jan Boehm ${ }^{\mathrm{a}}$, Mark Shortis ${ }^{\mathrm{b}}$ \\ ${ }^{a}$ Dept. Of Civil, Environmental and Geomatic Engineering, University College London, London

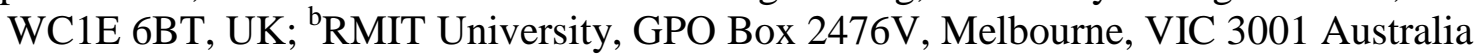

\begin{abstract}
Multi-View Stereo (MVS) as a low cost technique for precise 3D reconstruction can be a rival for laser scanners if the scale of the model is resolved. A fusion of stereo imaging equipment with photogrammetric bundle adjustment and MVS methods, known as photogrammetric MVS, can generate correctly scaled 3D models without using any known object distances. Although a huge number of stereo images (e.g. 200 high resolution images from a small object) captured of the object contains redundant data that allows detailed and accurate 3D reconstruction, the capture and processing time is increased when a vast amount of high resolution images are employed. Moreover, some parts of the object are often missing due to the lack of coverage of all areas. These problems demand a logical selection of the most suitable stereo camera views from the large image dataset. This paper presents a method for clustering and choosing optimal stereo or optionally single images from a large image dataset. The approach focusses on the two key steps of image clustering and iterative image selection. The method is developed within a software application called Imaging Network Designer (IND) and tested by the 3D recording of a gearbox and three metric reference objects. A comparison is made between IND and CMVS, which is a free package for selecting vantage images. The final 3D models obtained from the IND and CMVS approaches are compared with datasets generated with an MMDx Nikon Laser scanner. Results demonstrate that IND can provide a better image selection for MVS than CMVS in terms of surface coordinate uncertainty and completeness.
\end{abstract}

Keywords: Multi View Stereo (MVS), Structure from Motion (SfM), Close Range Photogrammetry, Imaging Network, Image Clustering and Selection

\section{INTRODUCTION}

Photogrammetric Multi-view Stereo method has proved its ability to be a serious rival for laser scanners for accurate and dense 3D reconstruction of both cultural heritage and industrial objects ${ }^{1,2} ;$. If stereo images are used, this method can resolve the scale without the need for any control targets or scale bars placed on or around the object ${ }^{2}$. Designing a good imaging network in the first step of this method plays a crucial role in an accurate and dense reconstruction ${ }^{3} ;{ }^{4} ;{ }^{5} ;{ }^{6}$. Although taking many images from different postures will deliver a strong imaging network, the elapsed time for capturing and processing the images will be considerably increased, especially in the dense 3D reconstruction step where stereo matching algorithms are exploited ${ }^{7} ;{ }^{8} ;{ }^{9}$. This issue proves the necessity of a step before dense matching for selecting the optimal images from the large image dataset ${ }^{10}$. Although in the previous work a new approach was proposed for this step ${ }^{1}$, the method was designed to select single images which could not be used for short base line stereo matching algorithms. Furthermore, the accuracy of the final point cloud was not checked due to the lack of reliable comparative dataset for each of the test objects.

This paper describes a novel approach for clustering and selecting optimal stereo images from an existing dense image dataset such that stereo matching of the selected images results in a complete point cloud with a level of predefined uncertainty. Following a review of related work in the next section, the approach is described in the methodology section and rigorously tested in the experiments and results sections.

\section{BACKGROUND}

The photogrammetric MVS workflow for accurate 3D reconstruction from stereo images can be outlined as follows:

a) Design of the stereo imaging configuration (stereo imaging network configuration, stereo sensor selection) ${ }^{11},{ }^{4},{ }^{12}$;

Videometrics, Range Imaging, and Applications XII; and Automated Visual Inspection, edited by Fabio Remondino,

Mark R. Shortis, Jürgen Beyerer, Fernando Puente León, Proc. of SPIE Vol. 8791, 879107

(C) 2013 SPIE · CCC code: 0277-786X/13/\$18 - doi: 10.1117/12.2020472 
b) Geometric and radiometric calibration of the stereo cameras ${ }^{13}$;

c) Image capture and removal of geometric distortions from the images ${ }^{13}$;

d) Accurate image measurements using Structure from Motion (SfM) methods ${ }^{14},{ }^{15},{ }^{16},{ }_{17},{ }^{18},{ }^{19},{ }^{20},{ }^{15}$;

e) Correct scaling and the improvement of accuracy using a robust photogrammetric bundle adjustment with external constraints ${ }^{21}$, ;

f) Image clustering and selection of images with the best content for reconstruction ${ }^{22}$;

g) Generation of a dense 3D point cloud with MVS methods ${ }^{7}, 23$;

h) Surface reconstruction and rendering ${ }^{24}$.

In order to cluster and select an optimal set of images (step f) able to meet a specified uncertainty and completeness, a method $^{1}$ was implemented within a software application called Imaging Network Designer (IND). The aim of the research was to extract the optimal image dataset for 3D reconstruction from an organized image dataset that has been systematically captured in a professional photographic studio by moving a camera around the cultural heritage object. The strategy selects key viewpoints for 3D reconstruction and removes redundant images. The selected images are then exploited in an MVS routine to produce an accurate, dense and colour-corrected 3D model. Selection methodology differed from that presented in ${ }^{25}$ by considering many parameters defined by ${ }^{3}$ and ${ }^{4}$ including: (1) field of view (FoV) as a common constraint between range-related and visibility-related constraints; (2) self-occluded areas and; (3) rangerelated constraints (including depth of field, field of view, resolution and scale constraints). The method improves upon that presented by ${ }^{26},{ }^{27}$ which, although designed as a rule-based strategy to find the minimum number of viewpoints within an optimal configuration that would provide sufficient coverage and accuracy for $3 \mathrm{D}$ reconstruction of cultural heritage objects, is unable to resolve self-occluded areas and relies upon stochastic methods which produce different results for each run. IND was compared with a well-known package, called CMVS ${ }^{10}$, which can extract a relevant image dataset from thousands of images downloaded from the internet. CMVS clusters and selects the optimal viewpoints through a four step iterative procedure including merging the sparse point cloud generated with Structure from Motion, removing low quality images, clustering images into smaller components using Normalized Cuts algorithm ${ }^{28}$ and finally adding images to weak clusters. Because CMVS was designed for extremely large photo collections (e.g. more than 10,000 photos uploaded to the internet), it ignores many metric constraints considered in photogrammetric network design. The previous work proved the advantages of IND in comparison with CMVS for selecting optimal images captured of cultural heritage objects. Some of drawbacks of our previous research are addressed in this paper including; a) optimization of the self-occluded test; b) single image selection instead of stereo image selection, and c) validation against reference data sets.

\section{METHODOLOGY}

Given a low quality mesh of the object and starting values generated as output from SfM, a relatively homogenous imaging network ${ }^{26}$, can be achieved by considering the visibility of each point on the surface. A four-zone cone is defined with its apex located on each surface point and an axis aligned to the surface point normal (Figure 1). A specific FoV (10 degrees) is considered as an acceptable viewing angle to each zone of this cone $\left(\mathrm{L}_{1-4}\right)$. Image clustering and image selection are performed based on the workflow mentioned in the next sections. The output of the image clustering step, a visibility matrix (Figure 1), allows selection of either optimal single or stereo images. 


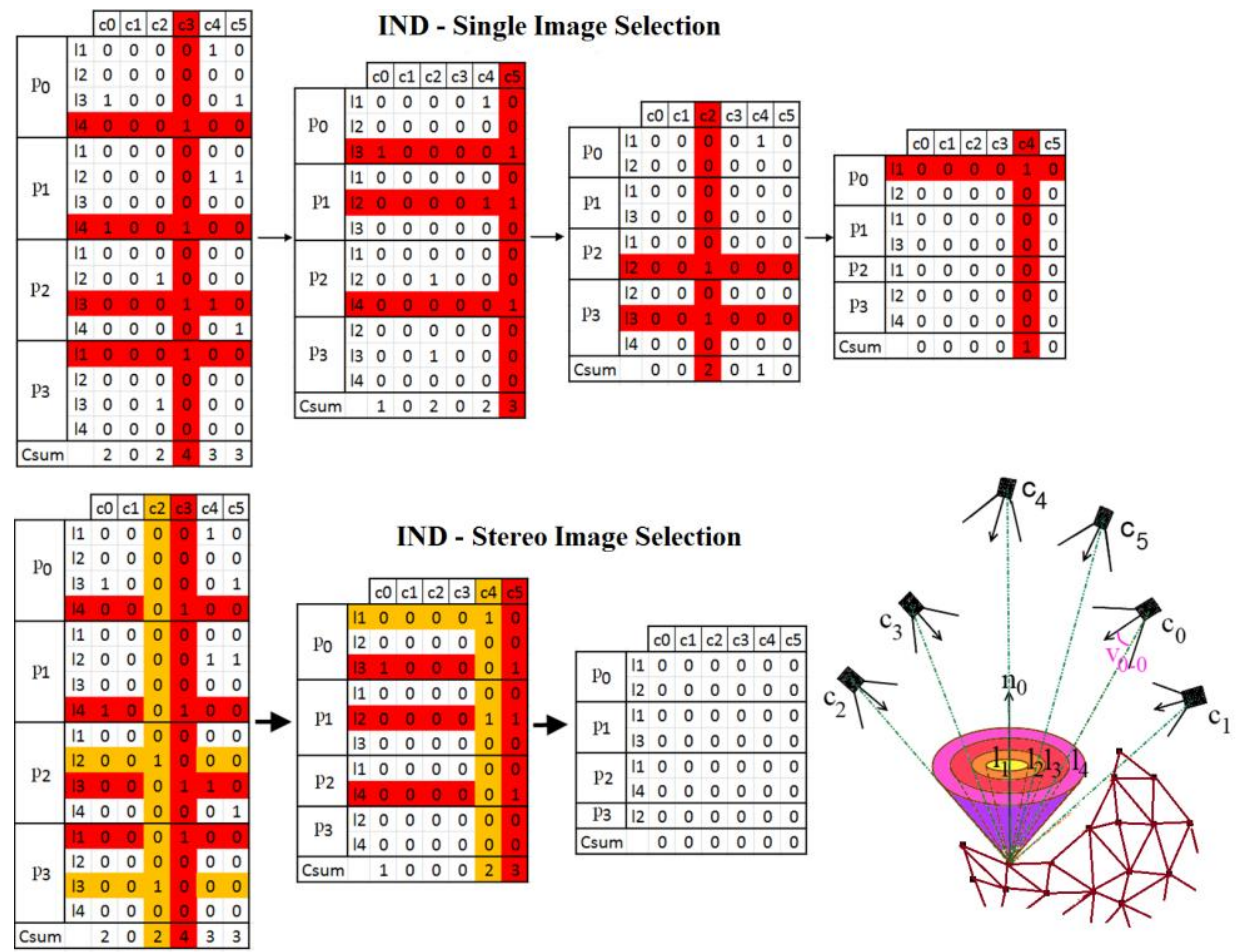

Figure 1. A surface point cone (right) and a schematic view of clustering and selection steps.

\subsection{Image Clustering}

In the first stage of image clustering, a boundary for the mesh is defined and maximum, minimum and optimal ranges for this object are calculated by using the approach cited in ${ }^{3}$ and ${ }^{4}$ which defines a set of functional constraints describing depth of field, field of view, resolution and image scale. Moreover, a visibility matrix is defined as a binary matrix with (row: $4 *$ number of points and column: number of viewpoints) and filled in based on the visibility and range related tests (Figure 2):

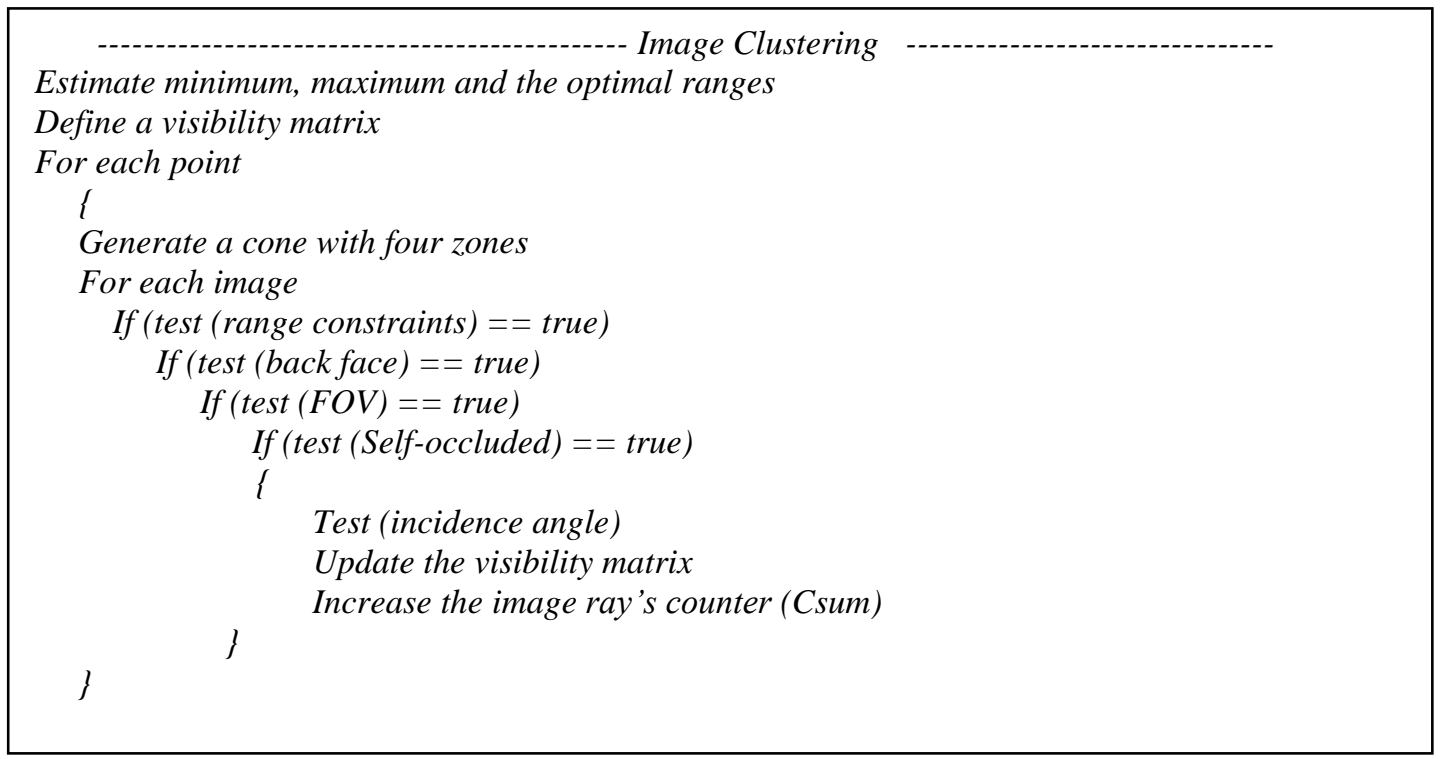

Figure 2. The pseudo code for image clustering in IND 


\subsubsection{Range-related constraints}

In order to check the range constraints for each surface point, the distance between each viewpoint and the surface point is compared with the maximum and minimum permissible ranges estimated in the first stage. If the calculated distance is located within these cut-off ranges, the viewpoint passes the range constraint.

\subsubsection{Visibility-related constraints}

These constraints are tested for each surface point of the rough mesh by taking into account surface points located on back faces and considering the self-occluded area, the field of view of each image and the incidence angle of the view to the surface point normal.

\subsubsection{Back face test}

A surface point is back facing if its normal points away from the camera. Therefore, if the dot product between the line of sight coming from the camera and surface normal is positive, the point is located in the back face ${ }^{29}$. Filtering the back face points in the first step of the visibility tests increases the speed of processing by ignoring the calculations of other tests for the surface points that fail this test.

\subsubsection{Self-occluded area}

A self-occluded area occurs when part of the object surface blocks the line of sight for another underlying area of the object from a specific viewpoint. A surface point is visible in the image if there are no entities between the camera and the point. The self-occluded constraint uses the following procedure:

a) Re-arranging the ordering of mesh triangles with regards to the distance to the test camera view.

b) For triangles closer to the camera than the surface point under test, finding the intersection between the line of sight from the camera and the plane of each triangle ${ }^{29}$. If the intersection point is inside the triangle ${ }^{30}$, the point is not visible and the point fails the test.

\subsubsection{Field of view constraint}

To make sure the surface point is located within the field of view of a camera, the angle between the ray coming from each camera to the point and the optical axis of the camera is computed (V in Figure 1). If this angle is less than half of the FoV, the viewpoint passes the FoV test.

\subsubsection{Incidence angle of the surface point}

The uncertainty in estimating the position of a surface point primarily depends on the triangulation angle (the smaller the angle, the higher uncertainty). However, a surface point cannot be distinctly recognized in an image if the camera is located at, or close to, the horizon of the surrounding surface. In order to consider these constraints, the visibility matrix (Figure 1 left) is updated. Having defined the cone of a given surface point, if a viewpoint satisfies all of the above constraints, and the line of sight is located in one of the zones of the surface point cone, the value of the zone in the visibility matrix will be set to one. Successive viewpoints are clustered for each surface point according to incidence angle, which is the angle between the line of sight from the camera and the negative direction of the surface point normal. This angle is compared with boundaries of each zone of the surface point cone (Figure 1 left, rows 11 to 14 in the visibility matrix) to allocate a value ( 0 or 1$)$ for the correspondent cell in the visibility matrix.

\subsection{Image Selection}

Having classified the viewpoints, the selected viewpoints should be reduced to an optimal number for 3D reconstruction. The process of selecting optimal viewpoints is illustrated in a schematic view in Figure 1 and the pseudo-code in Figure 3. This process differs from the method presented in ${ }^{1}$ by selecting image pairs rather than single images. For each image, simulated image observations are only generated with backward intersection using collinearity equations ${ }^{31}$ for those surface points that pass the range and visibility tests in the first step, denoted by non-zero values in the visibility matrix. In common with ${ }^{1}$, viewpoints are selected such that the number of surface points seen in each view is a maximum. The 3D coordinates and uncertainties of surface points with at least four image observations are computed with photogrammetric space intersection ${ }^{31}$. The image selection process is iteratively continued, adding image pairs to 
the network and eliminating them from the visibility matrix, until specified measurement uncertainty and completeness criteria are satisfied.

Coordinate Uncertainty: One of the priorities is to achieve a set of points with a given level of uncertainty. Uncertainty is expressed in relative terms through the Root Mean Square (RMS) uncertainty of the XYZ object point coordinates computed within the network adjustment ${ }^{21}$ divided by the maximum length of the object.

Completeness: In order to ensure dense 3D reconstruction, $90 \%$ of all surface points must be successfully coordinated.

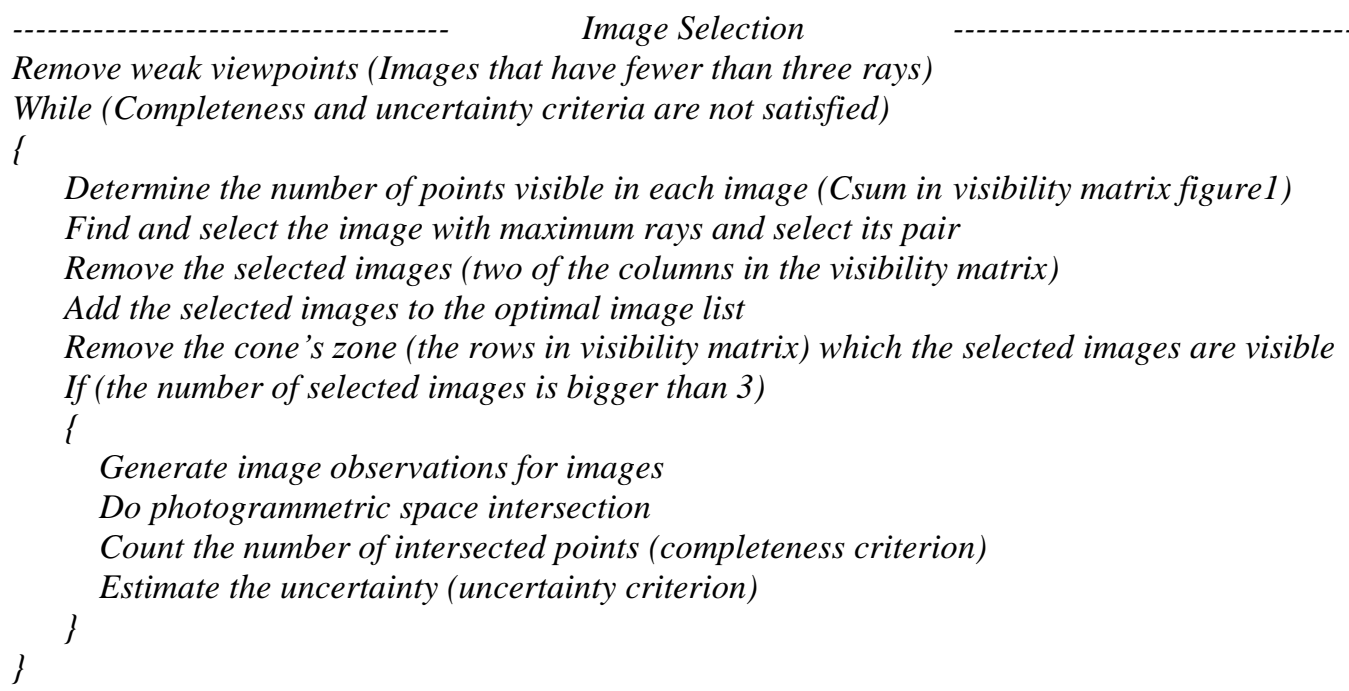

Figure 3. The pseudo code for stereo image selection in IND 


\section{EXPERIMENTS}

In order to evaluate the performance of the presented method, a set of geometric reference objects and a gearbox casing were imaged with two Nikon D700 digital SLR cameras. The cameras were mounted base plate to base plate using a purpose-manufactured adaptor to provide a minimal stereo base whilst allowing access to batteries and memory cards. Each D700 camera was equipped with a $35 \mathrm{~mm}$ fixed focal length lens imaging onto a 12 megapixel sensor (4256 $\times$ 2832) with $8.4 \mu \mathrm{m}$ square pixels.
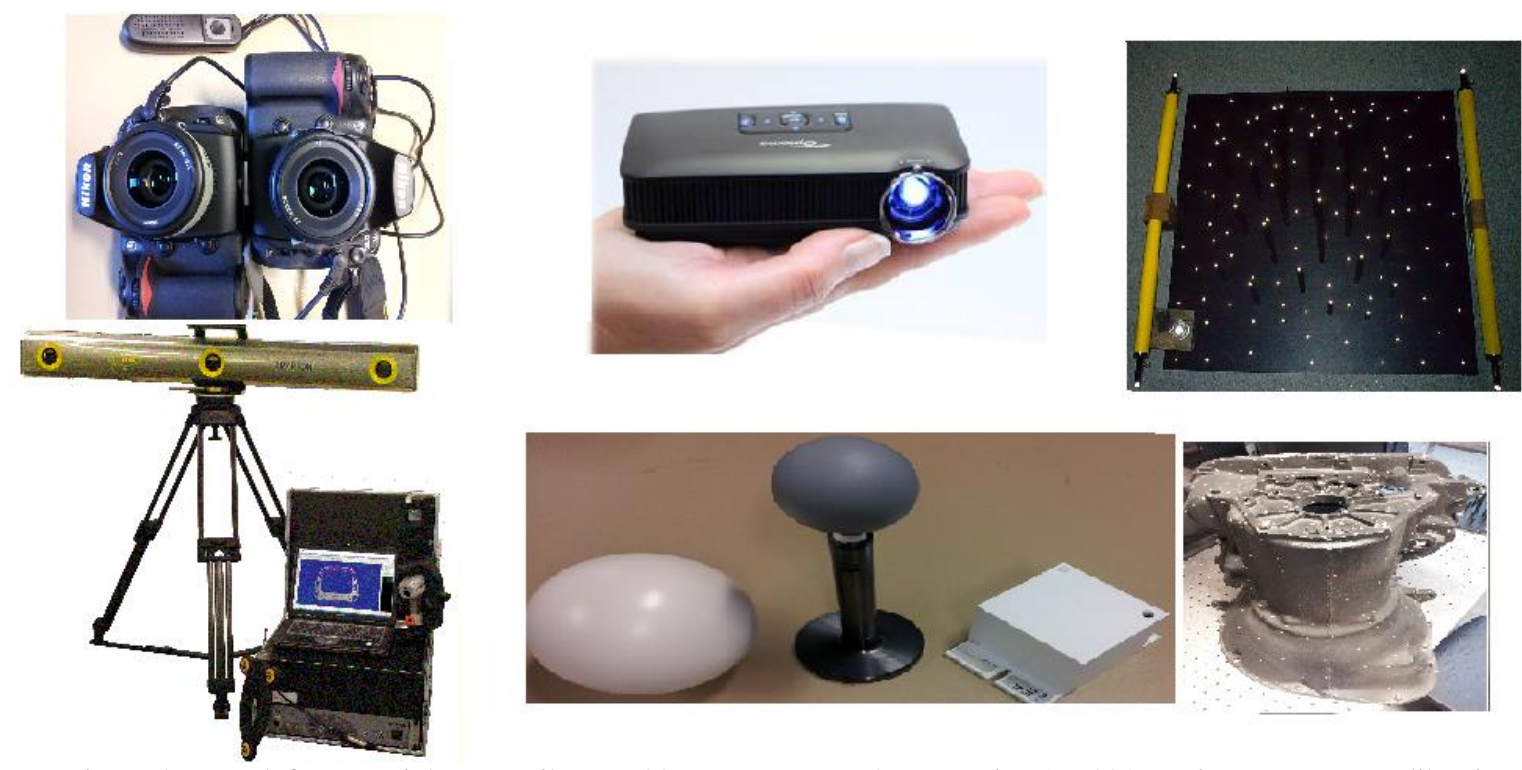

Figure 4. From left top to right: our Nikon D700 stereo camera, Optoma Pico (PK301) projector, camera calibration object with Brunson scale bars; from left bottom to right: Nikon MMDx laser scanner, large and small reference spheres, Nikon calibration cube and gearbox.

The cameras were calibrated with a 3D calibration fixture, equipped with 119 retro reflective targets mounted on vertical bars and a base plate $(550 \times 550 \mathrm{~mm}$ ) (Figure 4). Scale was provided by two $600 \mathrm{~mm}$ long Brunson carbon fibre scale bars equipped with retro-target tips. The scale bar lengths are known to a precision of $\pm 0.003 \mathrm{~mm}$. A $100 \mathrm{~mm}$ white Nikon reference cube with precisely known inter-plane separations was used to validate the Nikon MMDx laser scanner and also served as a reference object for evaluating the accuracy of the method. In addition to the cube, two reference spheres and a metal gearbox casting were used as test objects. Camera calibration parameters were estimated for both cameras to a high level of precision (mean precision of target coordinates was $\pm 5 \mu \mathrm{m}$ ). The self-calibrations were performed with Vision Measurement System $\left(\mathrm{VMS}^{32}\right.$ ) which was also used to compute relative orientations of the stereo cameras. The resultant standard deviation of estimated stereo base line $(102.201 \mathrm{~mm})$ was $\pm 85 \mu \mathrm{m}$.
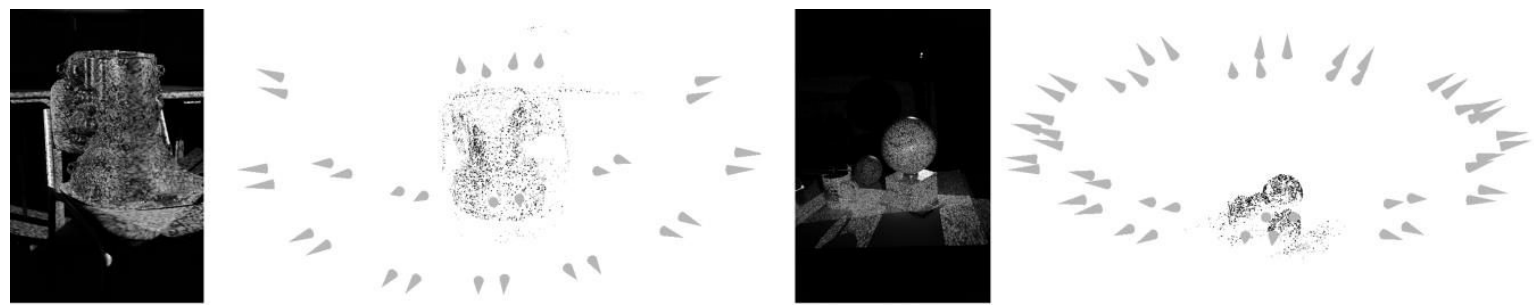

Figure 5. Example images of the textured gearbox and reference objects with Nikon stereo camera and the schematic imaging network for each group.

3D surface reconstruction based on dense image matching requires varying texture on the object surface. Since all of the objects used here had little to no discernible texture, two Optoma Pico (PK301) projectors were used to project a pattern onto the surfaces. This pattern was selected from stock pebble and gravel images to generate a fine 
and natural texture on the object. Strong convergent image networks (Figure 5) were captured with the stereo camera. All images were taken using a tripod to ensure a high level of image quality. Figure 3 gives an overview of the image networks including 30 and 48 images acquired for each object group.

Having determined the interior orientation parameters of the stereo cameras from self-calibration, all images were geometrically corrected and imported into Bundler ${ }^{33}{ }^{14}$ in order to automatically estimate exterior orientation starting values. Bundler and the included SIFT based operator were used as they provide a highly effective automatic solution without the need for 3D control points. Bundler output data was transformed into VMS compatible format in order to compute a bundle adjustment with the camera base line constraint. This solution provided high quality exterior orientation parameter estimates and a check on object coordinate precision. The mean $3 \mathrm{D}$ object coordinate precision for the gearbox example was $\pm 11 \mu \mathrm{m}$. Equivalent values for the cube and sphere reference object set was $\pm 32 \mu \mathrm{m}$.

A rough mesh was provided with the accurate sparse point cloud obtained from the previous step and imported into IND. Given a specified uncertainty and the mode of image selection (stereo or single); IND selected the optimal images for each dataset. The selected images with visibility data are imported into PMVS ${ }^{23} 34$ to generate a dense point cloud.

\section{RESULTS}

In order to test the relative precision of IND in both stereo and single image selection modes, the photogrammetric bundle adjustment implemented in VMS was applied on the simulated image observations, point locations and exterior orientation parameters obtained with IND. Figure 6 illustrates the error ellipsoids of the points for different imaging networks provided with IND in single and stereo image selection modes. With a specified uncertainty 1:5,000, IND selected 12 single images covering the entire object with relatively low level of precision $(1: 14,000)$. The same number of images was automatically selected in the stereo case (Figure 6 - A and B), with more precise results $(1: 15,000)$ due to a better configuration. Increasing the specified uncertainty to $1: 5,500$ led to the selection of more image pairs and subsequently better precision in stereo mode $(1: 18,000)$, but not in single image mode (Figure 6 -A). All 30 images were chosen in stereo mode when 1:6,000 uncertainty was defined for IND (Figure 6 F), while 21 single images were selected for the same level of uncertainty which resulted in a degraded relative network precision $(1: 21,000)$ (Figure $6 \mathrm{C}$ ). The difference between the specified uncertainty and the relative precision is that the former is estimated through space intersection, but the latter is the result of bundle adjustment, which gives a more complete estimate.

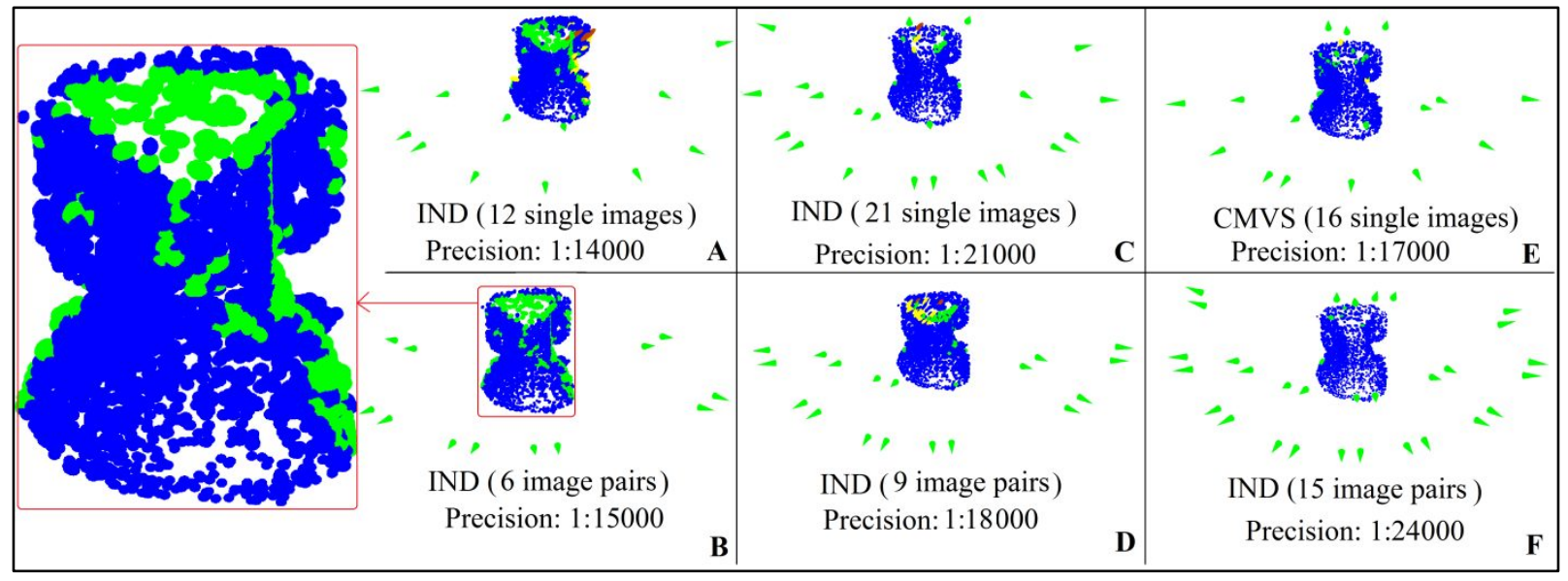

Figure 6. Error ellipsoids for different imaging networks provided with IND for gearbox. The green ellipsoids show the points with three rays, yellow are used for points with two rays, and the points visible in more than four images are painted blue. Red is showing the points rejected in bundle adjustment. 
The dense point cloud generated for each imaging network with PMVS was compared to a dataset collected with an optically tracked Nikon MMDx laser scanner. Whilst the CMVS imaging network (Figure 6 right) led to a relatively complete point cloud for the gearbox, the IND imaging networks (in both single and stereo image selection modes) with a specified uncertainty 1:6000 resulted in more complete point sets, especially in the flange of the gearbox (Figure 7). Moreover, there is no control in CMVS to specify the level of uncertainty or completeness. However, the precision of the CMVS point cloud can be determined by importing the CMVS image selection into IND and simulating image observations. The same bundle adjustment procedure can then be carried to estimate relative precision.

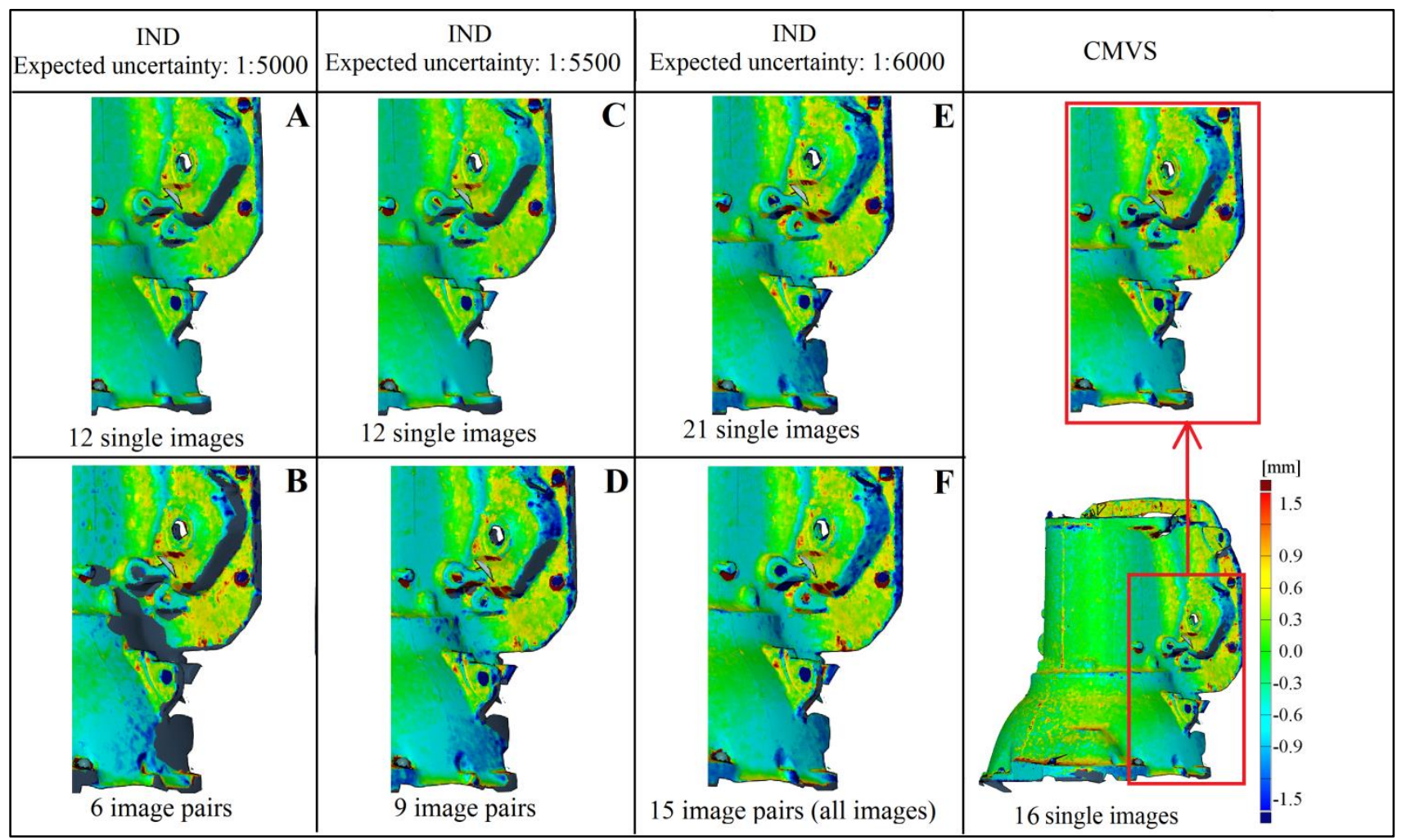

Figure 7. Comparison between laser scanner dataset and the mesh provided with photogrammetric Multi view stereo with using different image dataset generated with IND and CMVS for Gearbox.

In order to assess the ability of each method to determine optimal images from a superset of images captured in complete rings, another test was performed on the reference object dataset. PMVS could not generate a complete point cloud for the reference objects with the 17 images chosen with CMVS, as one side of the white cube, half of the small sphere and a quarter of the big sphere were completely missing. Given a specified uncertainty 1:5,000, IND selected 23 single images which could generate a relatively complete point cloud with PMVS. The network precision $(1: 9,000)$ for these images was lower than the precision estimated for 22 image pairs $(1: 13,000)$ selected with IND in stereo mode with the same specified uncertainty. Although importing all of the images in PMVS could produce a more complete point cloud, there were still some missing areas in the point cloud which showed that the imaging network designed for this project was not ideal. 


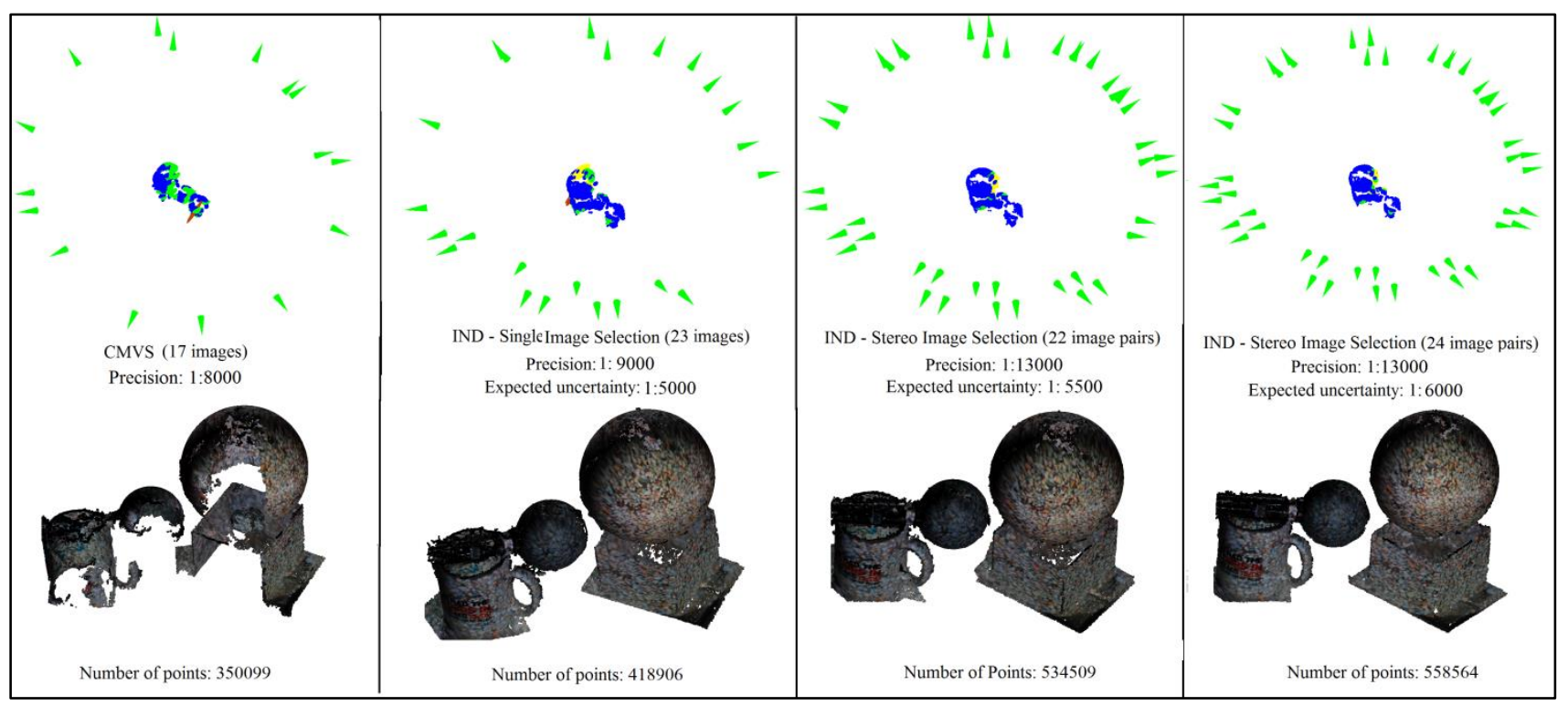

Figure 8. Uncertainty ellipsoids of the points for different imaging networks selected with IND and CMVS along with the point clouds generated for the reference object dataset on inputting the clustered sets of views into PMVS.

\section{CONCLUSION}

This paper described a significant improvement in the selection of optimal stereo images from a large image dataset intended for 3D object reconstruction. Our software platform (IND) considers visibility and range constraints to efficiently cluster and optimally select either stereo or single image sub-sets. Stereo image selection not only supports the use of short baseline stereo matching algorithms, which are extremely useful for dense point cloud generation of complex surfaces, but also utilizes the stereo base separation to provide scale throughout the measurement space. Comparing the point clouds obtained with images selected with IND and CMVS proved that IND could improve on image subset selection when compared with CMVS, which is a well-known and widely used package for 3D imaging. The reliability of IND in two different modes and CMVS was assessed by comparing the point clouds generated with images selected with each of these methods and the dataset provided with Nikon MMDx laser scanner. The majority of data points agreed with the laser scanner to $0.6 \mathrm{~mm}$ RMS. The next stage of this research project will present a strategy for automatically designing and delivering an optimal imaging network, which identifies the best camera positions for image capture around an artefact. This will decrease the number of images required for generating an accurate point cloud while ensuring optimal surface coverage and reconstruction.

\section{REFERENCES}

[1] Hosseininaveh, A., Serpico, M., Robson, S., Hess, M., Boehm, J., Pridden, I., and Amati, G., "Automatic Image Selection in Photogrammetric Multi-view Stereo Methods," Eurographics (EG), European Association for Computer Graphics9-16 (2012).

[2] Hosseininaveh, A., Robson, S., Boehm, J., Shortis, M., and Wenzel, K., "A Comparison of Dense Matching Algorithms for Scaled Surface Reconstruction Using Stereo Camera Rigs," ISPRS Journal of Photogrammetry and Remote Sensing 157-167 (2013).

[3] Mason, S., "Expert system-based design of close-range photogrammetric networks," ISPRS Journal of Photogrammetry and Remote Sensing 50(5), 13-24 (1995). 
[4] Saadatseresht, M., Fraser, C.S., Samadzadegan, F., and Azizi, A., "Visibility analysis in vision metrology network design," The Photogrammetric Record 19(107), 219-236 (2004).

[5] Saadatseresht, M., and Samadzadegan, F., "Multi-Objective Optimization of Vision Metrology Camera Placement Based on Pareto front Concept by NSGA-II Method," Journal of Faculty of Engineering (University of Tehran) 42 (3),313-326 (2008).

[6] Dunn, E., and Frahm, J.M., "Next best view planning for active model improvement," in Proceedings of British Machine Vision Conference (2009).

[7] Seitz, S.M., Curless, B., Diebel, J., Scharstein, D., and Szeliski, R., "A comparison and evaluation of multi-view stereo reconstruction algorithms," Computer Vision and Pattern Recognition, 2006 IEEE Computer Society, 1, 519-528 (2006).

[8] Hirschmüller, H., "Stereo processing by semiglobal matching and Mutual Information," IEEE Transactions on Pattern Analysis and Machine Intelligence 30(2), 328-341 (2008).

[9] Furukawa, Y., and Ponce, J., “Accurate, dense, and robust multi-view stereopsis," IEEE transactions on pattern analysis and machine intelligence1362-1376 (2009).

[10] Furukawa, Y., Curless, B., Seitz, S.M., and Szeliski, R., "Towards internet-scale multi-view stereo," in Computer Vision and Pattern Recognition (CVPR), 2010 IEEE, 1434-1441 (2010).

[11] Fraser, C.S., "Network design considerations for non-topographic photogrammetry," Photogrammetric Engineering and Remote Sensing 50(8), 1115-1126 (1984).

[12] Olague, G., and Dunn, E., "Development of a practical photogrammetric network design using evolutionary computing," The Photogrammetric Record 22(117), 22-38 (2007).

[13] Remondino, F., and Fraser, C., "Digital camera calibration methods: considerations and comparisons," International Archives of Photogrammetry, Remote Sensing and Spatial Information Sciences 36(5), 266-272 (2006).

[14] Snavely, N., Seitz, S.M., and Szeliski, R., "Photo tourism: exploring photo collections in 3D," in ACM SIGGRAPH 2006 Papers, 835-846 (2006).

[15] Agarwal, S., Snavely, N., Simon, I., Seitz, S.M., and Szeliski, R., "Building Rome in a day," IEEE Conference on Computer Vision (ICCV),72-79 (2009).

[16] Beardsley, P., Torr, P., and Zisserman, A., "3D model acquisition from extended image sequences," Computer Vision (ECCV),683-695 (1996).

[17] Pollefeys, M., Koch, R., and Gool, L.V., "Self-calibration and metric reconstruction inspite of varying and unknown intrinsic camera parameters," International Journal of Computer Vision 32(1), 7-25 (1999).

[18] Hao, X., and Mayer, H., "Orientation and auto-calibration of image triplets and sequences," International Archives Of Photogrammetry Remote Sensing And Spatial Information Sciences 34(3/W8), 73-78 (2003).

[19] Dick, A.R., Torr, P.H.S., and Cipolla, R., "Modelling and interpretation of architecture from several images," International Journal of Computer Vision 60(2), 111-134 (2004).

[20] Nister, D., "Automatic passive recovery of 3D from images and video," International Symposium on 3D Data Processing, Visualization and Transmission (3DPVT),438- 445 (2004).

[21] Atkinson, K.B., [Close range photogrammetry and machine vision], Whittles (2001).

[22] Furukawa, Y., Curless, B., Seitz, S.M., and Szeliski, R., "Towards internet-scale multi-view stereo" (2010).

[23] Furukawa, Y., and Ponce, J., “Accurate, Dense, and Robust Multiview Stereopsis,” IEEE Transactions on Pattern Analysis and Machine Intelligence 32(8), 1362-1376 (2010). 
[24] Remondino, F., and El-Hakim, S., "Image-based 3D Modelling: A Review," The Photogrammetric Record 21(115), 269-291 (2006).

[25] Hoppe, C., Wendel, A., Zollmann, S., Pirker, K., Irschara, A., Bischof, H., and Kluckner, S., "Photogrammetric Camera Network Design for Micro Aerial Vehicles," in Computer Vision Winter Workshop (CVWW), 8 (2012).

[26] Alsadik, B.S., Gerke, M., and Vosselman, G., "Optimal Camera Network Design for 3D Modeling of Cultural Heritage," in XXII ISPRS Congress 1-3 (2012).

[27] Alsadik, B., Gerke, M., and Vosselman, G., "Automated camera network design for 3D modeling of cultural heritage objects," Journal of Cultural Heritage (2012).

[28] Shi, J., and Malik, J., "Normalized cuts and image segmentation," IEEE Transactions on Pattern Analysis and Machine Intelligence 22(8), 888-905 (Aug).

[29] Hill, F.S., [Computer Graphics: Using OpenGL] , Prentice Hall (2001).

[30] Ericson, C., [Real-Time Collision Detection] , CRC Press (2004).

[31] Luhmann, T., Robson, S., Kyle, S., and Harley, I., [Close range photogrammetry: principles, techniques and applications], Whittles (2006).

[32] Shortis, M., and Stuart, R., [Vision Measurement System (VMS), http://www.geomsoft.com/VMS/] (2001).

[33] Bundler, [http://phototour.cs.washington.edu/bundler/], Washington University (2010).

[34] PMVS, [http://grail.cs.washington.edu/software/pmvs/], Washington University (2010). 